\title{
Boundary Value Problems of Nonlinear Variable Coefficient Fractional Differential Equations
}

\author{
Badawi Hamza Elbadawi Ibrahim ${ }^{1,3}$, Qixiang Dong ${ }^{2}$, Zhengdi Zhang ${ }^{1, *}$ \\ ${ }^{1}$ Department of Mathematics, Faculty of Science, Jiangsu University, Zhenjiang, China \\ ${ }^{2}$ School of Mathematical Sciences, Yangzhou University, Yangzhou, China \\ ${ }^{3}$ Department of Mathematics, Faculty of Education, University of Khartoum, Sudan
}

\section{Email address:}

badawi.12@hotmail.com (B. H. E. Ibrahim), dyzhang@ujs.edu.cn (Zhengdi Zhang)

${ }^{*}$ Corresponding author

\section{To cite this article:}

Badawi Hamza Elbadawi Ibrahim, Qixiang Dong, Zhengdi Zhang. Boundary Value Problems of Nonlinear Variable Coefficient Fractional Differential Equations. American Journal of Applied Mathematics. Vol. 7, No. 6, 2019, pp. 157-163. doi: 10.11648/j.ajam.20190706.13.

Received: October 24, 2019; Accepted: November 19, 2019; Published:Published: December 30, 2019

\begin{abstract}
It is recognized that the theory of boundary value problems for fractional order-differential equations is one of the rapidly developing branches of the general theory of differential equations. As far as we know, most of the papers studied the fractional Riemann-Liouville derivative with respect to boundary values that are zero. However, for the purpose of this study, we concern ourselves with Captou type derivative of the order $\alpha \in(2,3)$, with respect to boundary values that are nonzero. We establish sufficient conditions for the existence of solutions for boundary value problem of nonlinear variable coefficient of fractional order. On the other hand, the boundary value problem is formulated as follows:

$$
{ }^{c} D^{\alpha} u(t)+p(t) f(t, u(t))+q(t)=0, u(0)=a, u^{\prime}(0)=b, u(1)=d
$$

Where $a, b, d \in R$ are constants. In this paper, we investigate the existence and uniqueness of solutions for a class of boundary value problem of the nonlinear variable coefficient of fractional differential equations. The existence of solutions involving Captuo fractional derivatives is discussed under the assumption that the bounded conditions are constants. By means of the Banach contraction mapping principle and Larry- Schauder alternative, the existence of solutions are obtained. Finally, some examples are discussed to illustrate the results, which are generalized to nonlinear fractional derivatives with variable coefficients.
\end{abstract}

Keywords: Fractional Derivatives, Fixed Point Theorem, Boundary Value Problem

\section{Introduction}

In recent years, the theory of fractional differential equations has become an important area of study, see [1, $2,3,4]$. Boundary value problems of fractional differential equations have applications in various fields of science such as physics, mechanics, chemistry, engineering, etc. [7, 6, 5, 8].

\begin{abstract}
Also, it has received great attention and a variety of results concerning the existence of solutions, based on different kinds of analytic techniques, which can be found in $[11,10,9,12]$. In [13] the authors considered the existence of multiple positive solutions for the following fractional differential equations with a negatively perturbed term
\end{abstract}

$$
\left\{\begin{array}{l}
-D^{\alpha} u(t)=p(t) f(t, u(t))-q(t), \quad 0<t<1 \\
u(0)=u^{\prime}(0)=u(1)=0
\end{array}\right.
$$


Where $D^{\alpha}$ is the standard Riemann-Liouville derivative, $2<\alpha \leq 3$ is a real number, $q:(0,1) \rightarrow[0, \infty]$ is Lebesgue integrable and does not vanish identically on any subinterval of $(0,1)$. They established the existence results by Krasnoselskii's fixed point theorem in a cone. Cui [14] studied the following boundary value problem

$$
\left\{\begin{array}{l}
D^{p} x(t)+p(t) f(t, x(t))+q(t)=0, \quad 0<t<1, \\
x(0)=x^{\prime}(0)=0, \quad x(1)=0,
\end{array}\right.
$$

where $D^{p}$ is the standard Riemann-Liouville derivative, $2<$ $p \leq 3$ is a real number, $q:(0,1) \rightarrow R$ is continuous and Lebesgue integrable, $p:(0,1) \rightarrow[0, \infty)$ is continuous and does not vanish identically on any subinterval of $(0,1)$. Under the assumption that $f(t, x)$ is a Lipschitz continuous function, by using $u_{0}$-positive operator, he deduced that the Lipschitz constant is related to the first eigenvalues corresponding to the relevant operators.

In this paper we study the following boundary value problems for fractional differential equation (BVP in short)

$$
\left\{\begin{array}{l}
{ }^{c} D^{\alpha} u(t)+p(t) f(t, u(t))+q(t)=0, \quad 0<t<1, \\
u(0)=a, \quad u^{\prime}(0)=b, \quad u(1)=d,
\end{array}\right.
$$

where ${ }^{c} D^{\alpha}$ is Caputo fractional derivatives with $2<\alpha \leq 3$, $a, b, d \in R$ are constants, $q:(0,1) \rightarrow R$ is continuous and Lebesgue integrable and $p:(0,1) \rightarrow[0, \infty)$ is continuous and does not vanish identically on any subinterval of $(0,1)$.

\section{Preliminaries}

In this section, we state some definitions and results that we are going to use throughout this paper, we investigate the problem (1) with non-homogenous boundary value conditions. By using Banach contraction mapping principle and LarrySchauder alternative, the existence of solutions of the BVP (1) is obtained.

Definition 2.1. Let $p>0$ be a fixed number. The RiemannLiouville fractional integral of order $p>0$ of a function $h \in C([a, b])$ is defined by

$$
I_{a}^{p} h(t)=\frac{1}{\Gamma(p)} \int_{a}^{t}(t-s)^{p-1} h(s) d s, \quad t \in[a, b]
$$

provided the right side is point-wisely defined, where $\Gamma(\cdot)$ denotes the well-known gamma function, i.e., $\Gamma(z)=$ $\int_{0}^{\infty} \mathrm{e}^{-t} t^{z-1} \mathrm{~d} t$.

Definition 2.2. Let $p \geq 0$ and $n=[p]+1$. If $h \in A C^{n}[a, b]$ then the Caputo fractional derivative of order $p$ of $h$ at the point $t$ is defined by

${ }^{c} D_{a}^{p} h(t)=\frac{1}{\Gamma(n-p)} \int_{a}^{t}(t-s)^{n-p-1} h^{(n)}(s) \mathrm{d} s, \quad t \in[a, b]$
${ }^{c} D_{a}^{p}$ is also called the Caputo fractional differential operator.

For simplicity, when $a=0$, we denote ${ }^{c} D_{0}^{\alpha}$ and $I_{0}^{\alpha}$ by ${ }^{c} D^{\alpha}$ and $I^{\alpha}$, respectively.

Lemma 2.3. Let $p, q>0$ and $n=[p]+1$. Then the following relations hold.

$$
{ }^{c} D^{p} t^{q-1}=\frac{\Gamma(q)}{\Gamma(q-p)} t^{q-p-1}, q>n
$$

and

$$
{ }^{c} D^{p} t^{k}=0, k=0,1,2, \ldots \ldots, n-1 .
$$

Lemma 2.4. Let $p>0, h(t) \in C(0,1)$. The homogenous fractional differential equation

$$
{ }^{c} D^{\alpha} h(t)=0
$$

has a solution

$$
h(t)=c_{0}+c_{1} t+c_{2} t^{2}+\ldots \ldots+c_{n-1} t^{n-1},
$$

where $c_{i} \in R, i=0,1,2, \ldots, n-1$ are some constants.

Lemma 2.5. Let $\alpha, \beta \geq 0$ and $h \in L_{1}[a, b]$. Then $I^{\alpha} I^{\beta} h(t)=I^{\alpha+\beta} h(t)=I^{\beta} I^{\alpha} h(t)$ and ${ }^{c} D^{\alpha} I^{\alpha} h(t)=h(t)$ for all $t \in[a, b]$.

Lemma 2.6.(Leray-Schauder nonlinear alternative). Let $F$ be a Banach space and $\Omega$ a bounded open subset of $F, 0 \in \Omega$ and

$T: \bar{\Omega} \rightarrow F$ be a completely continuous operator. Then, either

(i)there exists $u \in \partial \Omega, \lambda>1$ such that $T(u)=\lambda u$, or

(ii) there exists a fixed point $u^{*} \in \bar{\Omega}$.

Lemma 2.7. Let $a, b, c \in R$ and $y \in C[0,1]$. The unique solution of the boundary value problem

$$
\left\{\begin{array}{l}
{ }^{c} D^{\alpha} u(t)+y(t)=0, \quad 0<t<1 \\
u(0)=a, \quad u^{\prime}(0)=b, \quad u(1)=d
\end{array}\right.
$$

is given by

$$
u(t)=h(t)+\int_{0}^{1} G(t, s) y(s) d s
$$

where $h(t)=(d-b-a) t^{2}+b t+a$, and $G(t, s)$ is the Green's function given by

$$
G(t, s)=\frac{1}{\Gamma(\alpha)} \begin{cases}t^{2}(1-s)^{\alpha-1}-(t-s)^{\alpha-1}, & 0 \leq s \leq t \leq 1 \\ t^{2}(1-s)^{\alpha-1}, & 0 \leq t \leq s \leq 1\end{cases}
$$


Proof. Applying Lemma 2.4 and 2.5, the Eq (2) is equivalent to the integral equation

$$
u(t)=-\frac{1}{\Gamma(\alpha)} \int_{0}^{t}(t-s)^{\alpha-1} y(s) d s-c_{0}-c_{1} t-c_{2} t^{2}
$$

The boundary condition $u(0)=a$, gives $c_{0}=-a$. Differentiating (5), we get

$$
u^{\prime}(t)=-\frac{1}{\Gamma(\alpha-1)} \int_{0}^{t}(t-s)^{\alpha-2} y(s) d s-c_{1}-2 c_{2} t .
$$

Since $u^{\prime}(0)=b$, we deduce that $c_{1}=-b$. Further, condition $u(1)=d$ implies that

$$
c_{2}=a+b-d-\frac{1}{\Gamma(\alpha)} \int_{0}^{1}(1-s)^{\alpha-1} y(s) d s
$$

Substituting $c_{0}, c_{1}, c_{2}$ into Eq (5), we obtain

or

$$
u(t)=(d-b-a) t^{2}+b t+a+\frac{1}{\Gamma(\alpha)} \int_{0}^{1} t^{2}(1-s)^{\alpha-1} y(s) d s-\frac{1}{\Gamma(\alpha)} \int_{0}^{t}(t-s)^{\alpha-1} y(s) d s
$$

$$
u(t)=(d-b-a) t^{2}+b t+a+t^{2} I^{\alpha} y(1)-I^{\alpha} y(t)
$$

Thus we get

$$
u(t)=h(t)+\frac{1}{\Gamma(\alpha)} \int_{0}^{1} G(t, s) y(s) d s
$$

The proof is completed.

\section{Existence Results}

In this section, Now we study the existence of solutions to BVP (1). Let us denote by $C([a, b], R)$ the Banach space of all continuous functions $u:[0,1] \rightarrow R$ endowed with supremum norm $\|u\|=\max _{t \in[0,1]}|u(t)|$.

Definition 3.1. A function $u:[0,1] \rightarrow R$ is said to be a solution to (1), if u satisfies

$$
u(t)=h(t)+\int_{0}^{1} G(t, s)[p(s) f(s, u(s))+q(s)] d s
$$

for $t \in[0,1]$.

Define an operator $T: C([0,1], R) \rightarrow C([0,1], R)$ by

$$
T u(t)=h(t)+\int_{0}^{1} G(t, s)[p(s) f(s, u(s))+q(s)] d s
$$

for $u \in C([0,1], R)$ and $t \in[0,1]$. Then we transform the existence of solutions to the fixed point problem. We first list the following hypothesis.

(H1) $f:[0,1] \times R \rightarrow R$ is continuous.

(H2) There exist nonnegative function $g \in L^{1}\left([0,1], R_{+}\right)$such that

$$
|f(t, u)-f(t, v)| \leq g(t)|u-v|
$$

for all $u, v \in R$ and $t \in[0,1]$.

(H3) There exist nonnegative function $\phi \in L^{1}\left([0,1], R_{+}\right)$, and a continuous nondecreasing function $\psi:[0, \infty) \rightarrow(0, \infty)$ such that

$$
|f(t, u)| \leq \phi(t) \psi(|u|)
$$


for all $(t, u) \in[0,1] \times R$.

Lemma 3.2. Let $\alpha>0$ and $f \in L_{1}\left([a, b], R_{+}\right)$, Then for all $t \in[a, b]$, we have

$$
I_{a}^{\alpha+1} f(t) \leq\left\|I_{a}^{\alpha} f\right\|_{L_{1}} .
$$

Proof. Let $f \in L_{1}\left([a, b], R_{+}\right)$. Then

$$
\begin{aligned}
\left\|I_{a}^{\alpha} f\right\|_{L_{1}} & =\int_{0}^{1} I^{\alpha} f(r) d r \geq \frac{1}{\Gamma(\alpha)} \int_{a}^{t} \int_{a}^{r}(r-s)^{\alpha-1} f(s) d s d r \\
& =\frac{1}{\Gamma(\alpha)} \int_{a}^{t}\left(\int_{s}^{t}(r-s)^{\alpha-1} f(s) d r\right) d s \\
& =\frac{1}{\alpha \Gamma(\alpha)} \int_{a}^{t}(t-s)^{\alpha} f(s) d s=I_{a}^{\alpha+1} f(t) .
\end{aligned}
$$

Theorem 3.3.Suppose that the condition (H1) and (H2) are satisfied. If

$$
N<1
$$

then the BVP (1) has a unique solution in $C([0,1], R)$, where $N=2\left\|I^{\alpha-1} p g\right\|$.

Proof. Define an operator $T: C([0,1], R) \rightarrow C([0,1], R)$ by

$$
T u(t)=h(t)+\int_{0}^{1} G(t, s)[p(s) f(s, u(s))+q(s)] d s
$$

for $u \in C([0,1], R)$ and $t \in[0,1]$. Then $u \in C([0,1], R)$ is a solution to the BVP (1) if and only if $u$ is a fixed point of $T$.

For $u, v \in C([0,1], R)$, applying (6), we obtain

$$
\begin{aligned}
|T u(t)-T v(t)| \leq & \int_{0}^{1} G(t, s)|p(s)[f(s, u(s))-f(s, v(s))]| d s \\
& =\left[I^{\alpha} p(1)(f(1, u(1))-f(1, v(1))]-\left[I^{\alpha} p(t)(f(t, u(t))-f(t, v(t))] .\right.\right.
\end{aligned}
$$

The condition (H2) implies that

$$
|T u(t)-T v(t)| \leq \max _{0 \leq t \leq 1}|u(t)-v(t)|\left[t^{2} I^{\alpha}|p(1)| g(1)+I^{\alpha}|p(t)| g(t)\right] .
$$

It follows from Lemma 3.2 that

$$
\|T u-T v\| \leq\|u-v\|\left[\left\|I^{\alpha-1} p g\right\|_{L_{1}}+\left\|I^{\alpha-1} p g\right\|_{L_{1}}\right]=2\left\|I^{\alpha-1} p g\right\|\|u-v\|
$$

For $t \in[0,1]$. Hence

$$
\|T u-T v\| \leq N\|u-v\| .
$$

The assumption (8) shows that $T$ is a contraction. By Banach contraction principle, $T$ has a unique fixed point in $C([0,1], R)$, which is the solution to the BVP (1). The proof is completed.

Next, we prove an existence result by using LarrySchouder's nonlinear alternative. For simplicity, let $k=$ $\max _{0 \leq t \leq 1}|h(t)|=|(d-b-a)+b+a|=|d|$, $M_{1}=2\left\|I^{\alpha-1} p \phi\right\|_{L_{1}}, M_{2}=2\left\|I^{\alpha-1} q\right\|_{L_{1}}+k$, and $M=$ $\max \left\{M_{1}, M_{2}\right\}$, where $\phi$ is the function appearing in condition
(H3).

Theorem 3.4. Suppose that $(\mathrm{H} 1)$ and $(\mathrm{H} 3)$ are satisfied. If

$$
\limsup _{r \rightarrow+\infty} M \frac{\psi(r)}{r}<1
$$

then the BVP (1) has at least one solution in $C([0,1], R)$.

Proof. First let us prove that $T$ is completely continuous. It is clear that $T$ is continuous since $f$ and $G$ are continuous. Since $\lim \sup _{r \rightarrow+\infty} M \frac{\psi(r)}{r}<1$, there exists a number $r>0$ such that $M(\psi(r)+1)<r$. Let $B_{r}=\{u \in C([0,1], R)$ : $\|u\| \leq r\}$. Then $B_{r}$ is a bounded subset in $C([0,1], R)$. For any $u \in B_{r}$, we have 


$$
\begin{aligned}
|T u(t)| & =\mid \int_{0}^{1} G(t, s)[p(s) f(s, u(s)+q(s)] d s+h(t) \mid \\
& \leq \int_{0}^{1}|G(t, s)|[|p(s)| \phi(s) \psi(\|u\|)+|q(s)|]+|h(t)| \\
& \leq \psi(r) \int_{0}^{1}|G(t, s)| d s|p(s)| \phi(s)+\int_{0}^{1}|G(t, s)| d s|q(s)|+|h(t)| \\
& \leq \psi(r)\left[I^{\alpha}|p(1)| \phi(1)+I^{\alpha}|p(s)| \phi(s)\right]+I^{\alpha}|q(1)|+I^{\alpha}|q(s)|+|h(t)| \\
& \leq \psi(r)\left[\left\|I^{\alpha-1} p \phi\right\|_{L^{1}}+\left\|I^{\alpha-1} p \phi\right\|_{L^{1}}\right]+\left\|I^{\alpha-1} q\right\|_{L^{1}}+\left\|I^{\alpha-1} q\right\|_{L^{1}}+k \\
& \leq \psi(r) 2\left\|I^{\alpha-1} p \phi\right\|_{L^{1}}+2\left\|I^{\alpha-1} q\right\|_{L^{1}}+k \\
& \leq\left(\psi(r) M_{1}+M_{2}\right) \leq M(\psi(r)+1)
\end{aligned}
$$

Hence $T\left(B_{r}\right)$ is uniformly bounded. For all $t_{1}, t_{2} \in[0,1], t_{1}<t_{2}$ and $u \in B_{r}$, we have,

$$
\begin{aligned}
\left|T u\left(t_{1}\right)-T u\left(t_{2}\right)\right| & \leq \int_{0}^{1}\left|G\left(t_{1}, s\right)-G\left(t_{2}, s\right)\right||p(s) f(s, u(s))| d s+\left|h\left(t_{1}\right)-h\left(t_{2}\right)\right| \\
& \leq \int_{0}^{1}\left|G\left(t_{1}, s\right)-G\left(t_{2}, s\right)\right||p(s)| \phi(s) \psi(\|u\|)|d s+| h\left(t_{1}\right)-h\left(t_{2}\right) \mid \\
& \leq \frac{\|p\| \psi(r)}{\Gamma(\alpha)}\left[\int_{0}^{t_{1}}\left|G\left(t_{1}, s\right)-G\left(t_{2}, s\right)\right| \phi(s) d s+\int_{t_{1}}^{t_{2}}\left|G\left(t_{1}, s\right)-G\left(t_{2}, s\right)\right| \phi(s) d s\right. \\
& \left.+\int_{t_{2}}^{1}\left|G\left(t_{1}, s\right)-G\left(t_{2}, s\right)\right| \phi(s) d s\right]+\left|h\left(t_{1}\right)-h\left(t_{2}\right)\right| \\
& \leq \frac{\|p\| \psi(r)}{\Gamma(\alpha)}\left[\int_{0}^{t_{1}}\left|t_{1}^{2}(1-s)^{\alpha-1}-\left(t_{1}-s\right)^{\alpha-1}-t_{2}^{2}(1-s)^{\alpha-1}+\left(t_{2}-s\right)^{\alpha-1}\right| d s\right. \\
& +\int_{t_{1}}^{t_{2}}\left|t_{1}^{2}(1-s)^{\alpha-1}-t_{2}^{2}(1-s)^{\alpha-1}+\left(t_{2}-s\right)^{\alpha-1}\right| d s \\
& \left.+\int_{t_{2}}^{1}\left|t_{1}^{2}(1-s)^{\alpha-1}-t_{2}^{2}(1-s)^{\alpha-1}\right| d s\right]+\left|h\left(t_{1}\right)-h\left(t_{2}\right)\right| \\
& \leq \frac{\|p\| \psi(r)}{\Gamma(\alpha)} \int_{0}^{t_{1}}\left[\left|t_{1}^{2}-t_{2}^{2}\right|(1-s)^{\alpha-1}+\left(t_{2}-s\right)^{\alpha-1}-\left(t_{1}-s\right)^{\alpha-1} d s\right. \\
& +\int_{t_{1}}^{t_{2}}\left|t_{1}^{2}-t_{2}^{2}\right|(1-s)^{\alpha-1}+\left(t_{2}-s\right)^{\alpha-1} d s \\
& \left.+\int_{t_{2}}^{1}\left|t_{1}^{2}-t_{2}^{2}\right|(1-s)^{\alpha-1}\right] d s+\left|h\left(t_{1}\right)-h\left(t_{2}\right)\right| \\
& \leq \frac{\|p\| \psi(r)}{\Gamma(\alpha+1)}\left[\left|t_{1}^{2}-t_{2}^{2}\right|\left(1-\left|1-t_{1}\right|^{\alpha}\right)+\left|t_{2}^{\alpha}-t_{1}^{\alpha}\right|-\left|t_{2}-t_{1}\right|^{\alpha}\right. \\
& +\left|t_{1}^{2}-t_{2}^{2}\right|\left(\left|1-t_{1}\right|^{\alpha}-\left|1-t_{2}\right|^{\alpha}\right)+\left|t_{2}-t_{1}\right|^{\alpha} \\
& \left.+\left|t_{1}^{2}-t_{2}^{2}\right|\left|1-t_{2}\right|^{\alpha}\right]+\left|h\left(t_{1}\right)-h\left(t_{2}\right)\right| . \\
&
\end{aligned}
$$

It follows that

$$
\left|T u\left(t_{1}\right)-T u\left(t_{2}\right)\right| \leq \frac{\|p\| \psi(r)}{\Gamma(\alpha+1)}\left[\left|t_{1}^{2}-t_{2}^{2}\right|+\left|t_{2}^{\alpha}-t_{1}^{\alpha}\right|\right]+\left|h\left(t_{1}\right)-h\left(t_{2}\right)\right|
$$


It is easy to see that $\left|T u\left(t_{1}\right)-T u\left(t_{2}\right)\right|$ tend to 0 as $t_{1}-t_{2} \rightarrow$ 0 , and the convergence is independent of $u \in B_{r}$. This show that $T\left(B_{r}\right)$ is equicontinuous. By Arzela- Ascolli Theorem we deduce that $T$ is completely continuous.

Now let $\Omega=\{u \in B:\|u\|<r\}$. Then $\Omega$ is an open and bounded subset in $B$ and $0 \in \Omega$. If there is a $u \in \partial \Omega$ such that $u=\lambda T u$ for some $\lambda \in(0,1)$ and each $t \in[0,1]$, then we have

$$
|u(t)|=\lambda|T u(t)| \leq|T u(t)| \leq M(\psi(r)+1)<r .
$$

This is contradictory to the fact that $u \in \partial \Omega$. Hence Lemma 2.6 (Leray-Schauder nonlinear alternative) allows us to conclude that $T$ has a fixed point $u^{*} \in \bar{\Omega}$. Therefore the BVP (1) has at least a solution $u^{*} \in B$. This completes the proof.

\section{Example}

Example 4.1. Consider the following fractional boundary value problem

$$
\left\{\begin{array}{l}
{ }^{c} D^{\frac{11}{4}} u(t)=\Gamma\left(\frac{11}{4}\right)\left[\frac{\frac{t^{2}}{5} u+t+3}{(1-t)^{\frac{3}{4}}}+\frac{1}{(1+t)}\right], \quad 0<t<1 \\
u(0)=a, \quad u^{\prime}(0)=b, \quad u(1)=d
\end{array}\right.
$$

In this case we have

$$
\begin{gathered}
f(t, x)=\frac{t^{2}}{5} u+t+3,2<\alpha=\frac{11}{4}<3 \\
p(t)=\frac{\Gamma\left(\frac{11}{4}\right)}{(1-t)^{\frac{3}{4}}}, q(t)=\frac{\Gamma\left(\frac{11}{4}\right)}{(1+t)} . \\
|f(t, x)-f(t, y)| \leq \frac{t^{2}}{5}|x-y|
\end{gathered}
$$

therefore

$$
|f(t, x)-f(t, y)| \leq g(t)|x-y|, \forall x, y \in R
$$

and

$$
\begin{gathered}
g(t)=\frac{t^{2}}{5} \\
\left\|I^{\alpha-1} p g\right\|_{L^{1}}=I^{\alpha} p(t) g(t)=\frac{1}{\Gamma(\alpha)} \int_{0}^{1}(1-s)^{\alpha-1} p(s) g(s) d s=\frac{1}{5} \int_{0}^{1}(1-s) s^{2} d s=\frac{\Gamma(2) \Gamma(3)}{5 \Gamma(5)}=0.01667<1 .
\end{gathered}
$$

Example 4.2. Consider the following problem

$$
\left\{\begin{array}{l}
{ }^{c} D^{\frac{7}{3}} u(t)=\frac{1}{\sqrt{1+t}} \frac{\left(1+t^{3}\right) e^{-t} u^{3}}{216}+\frac{t^{2}}{(1-t)}, \quad 0<t<1, \\
u(0)=a, u^{\prime}(0)=b, \quad u(1)=d,
\end{array}\right.
$$

has at least one solution. Applying Theorem (3.4), we have $\alpha=\frac{7}{3}$ and

$$
\begin{aligned}
|f(t, x)| & =\frac{1}{\sqrt{1+t}} \frac{\left(1+t^{3}\right) e^{-t} x^{3}}{216}+\frac{t^{2}}{(1-t)} \\
& \leq \frac{1}{\sqrt{1+t}}\left(1+t^{3}\right)\left(\frac{|x|}{6}\right)^{3}+\frac{t^{2}}{(1-t)} \\
& \leq p(t) \phi(t) \psi(|x|)+q(t) .
\end{aligned}
$$

where $p(t)=\frac{1}{\sqrt{1+t}}, \phi(t)=\left(1+t^{3}\right), \psi(|x|)=\left(\frac{|x|}{6}\right)^{3}, q(t)=\frac{t^{2}}{(1-t)}$.

Let us evaluate $M(\psi(r)+1)$, some computations lead to

$$
\begin{aligned}
\left\|I^{\alpha-1} p \phi\right\|_{L^{1}} & =I^{\alpha} p(t) \phi(t)=\frac{1}{\Gamma(\alpha)} \int_{0}^{1}(1-s)^{\alpha-1} p(s) \phi(s) d s=\frac{1}{\Gamma\left(\frac{7}{3}\right)} \int_{0}^{1}(1-s)^{\frac{4}{3}} \frac{1}{(1+s)^{\frac{1}{2}}}\left(1+s^{3}\right) d s \\
& \leq \frac{2}{\Gamma\left(\frac{7}{3}\right)} \int_{0}^{1}(1-s)^{\frac{4}{3}} d s \leq 0.7199
\end{aligned}
$$


And

$$
\left\|I^{\alpha-1} q\right\|_{L^{1}}=\frac{1}{\Gamma\left(\frac{7}{3}\right)} \int_{0}^{1}(1-s)^{\frac{4}{3}} \frac{s^{2}}{(1-s)} d s=\frac{\Gamma\left(\frac{4}{3}\right) \Gamma(3)}{\Gamma\left(\frac{7}{3}\right) \Gamma\left(\frac{13}{3}\right)}=0.2223 .
$$

In fact

$$
h(t)=(d-b-a) t^{2}+b t+a
$$

we take $a=b=d=0$, there for

$$
\begin{gathered}
k=|h(t)|=0, M_{1}=2\left\|I^{\alpha-1} p \phi\right\|_{L_{1}}=1.4398 \\
M_{2}=2\left\|I^{\alpha-1} q\right\|_{L^{1}}+k=0.4446, \text { then } M=1.8844
\end{gathered}
$$

we see that $(H 4)$ is equivalent to

$$
1.8844\left(\left(\frac{r}{6}\right)^{3}+1\right)-r<0 \text { forr }=2
$$

\section{Conclusion}

In this work we studied the existence and uniqueness of solutions for a class of boundary value problem of nonlinear variable coefficient of fractional differential equations. Employing Banach contraction theorem and LarrySchauder alternative, the existence of solutions were obtained. Some examples were discussed to illustrate our results which generalized to nonlinear Fractional derivatives with variable coefficients.

\section{Funding}

This work was supported by Key Program of the National Natural Science Foundation of China (Grant No. 11872189) and the National Natural Science Foundation of China (Grant No. 11472116).

\section{References}

[1] Miller. KS. Ross. B, An Introductionto the Fractional Calculusand Fractional Differential Equations. Wiley, NewYork, (1993).

[2] I. Podlubny, Fractional Differential Equations, Mathematics in Science and Engineering, Academic press, New York, 1999.

[3] R. Hilfer(Ed.), Applications of Fractional Calculus in Physics, World Scientific publishing Co, Singapore, 2000.

[4] K. Diethelm, The analysis of fractional differential equations, Lecture Notes in Mathmatics 2004, Springer-Verlag, Berlin, 2010.

[5] K. Diethelm, A. D. Freed, On the solution of nonlinear fractional-order differential equations used in the modeling of viscoplasticity, Sci. Comput. Chem. Eng. II, Springer Berlin Heidelberg, (1999), 217-224.

[6] M. A. Krasnoselskii, Two remarks on the method of successive approximations, (Russian) Uspehi Mat. Nauk (N.S.), 10(1955), 123-127.

[7] N. Heymans, I. Podlubny, Physical interpretation of initial conditions for fractional differential equations with RiemannLiouville fractional derivatives, Rheol. Acta, 45(2006), 765772.

[8] F. Metzler, W. Schick, H. G. Kilian, T. F. Nonnenmacher, Relaxation in filled polymers. A fractional calculus approach, J. Chem. Phys, 103(1995), 7180-7186.

[9] Ge. F, Kou. C, Stability analysis by Krasnoselskiis fixed point theorem for nonlinear fractional differential equations. Applied Mathematics and Computation 257(2015), 308-316.

[10] Su. X, Zhang. S. Unbounded solutions to aboundary value problem of fractional order on the half-line. Computers and Mathematics with Applications 61(2011), 1079-1087.

[11] Wang. G. Explicit iteration and unbounded solutions for fractional integral boundary value problem on an infinite interval. Applied Mathematics Letters 47(2015), 1-7.

[12] Yang. L. Application of Avery-Peterson fixed point theorem to nonlinear boundary value problem of fractional differential equation with the Caputos derivative. Communications in Nonlinear Science and Numerical Simulation 17 (2012), 45764584 .

[13] X. Zhang, L. Liu, Y. Wu, The Multiple positive solution of a singular fractional differential equations with negatively perturbed term, Math. Comput. Modelling 55(2012), 12631274.

[14] Y. Cui. Uniqueness of solution for boundary value problems for fractional differetial equation J. Appl. Math. Letters. 51(2016), 48-54. 
[15] A. A. Kilbas, H. M. Srivastava, J. J. Trujillo, Theory and applications of fractional differential equations, North-Holland Math. Stud., vol 204, Elsevier Science B.V., Amsterdam, 2006.
[16] K. Deimling, Nonlinear Functional Analysis, Springer-Verlag, Berlin, 1985. 